\title{
Validación de FACIT-TS-PS en una muestra de pacientes mexicanos con cáncer
}

\author{
Jazmín E. Hernández-Marín, ${ }^{1}$ Oscar Galindo-Vázquez, ${ }^{2 *}$ Rosario Costas-Muñíz, ${ }^{3}$ \\ Paula Cabrera-Galeana, ${ }^{4}$ M. del Rosario Caballero-Tinoco, ${ }^{5}$ José L. Aguilar-Ponce ${ }^{6}$ y Abel Lerma ${ }^{7}$ \\ ${ }^{1}$ Universidad Nacional Autónoma de México, Facultad de Psicología; ${ }^{2}$ Instituto Nacional de Cancerología, Servicio de Psicooncología; ${ }^{3}$ Memorial \\ Sloan-Kettering Cancer Center, Departamento de Psiquiatría y Ciencias del Comportamiento, Nueva York, Estados Unidos; ${ }^{4}$ Instituto Nacional de \\ Cancerología, Jefatura de Oncología Médica; 5 Instituto Nacional de Cancerología, Servicio de Enfermería; ${ }^{6}$ Instituto Nacional de Cancerología, \\ Oncología Médica; ${ }^{7}$ Universidad Autónoma del Estado de Hidalgo, Instituto de Ciencias de la Salud, Hidalgo, México. 1,2,4,5,6Ciudad de México, México
}

\section{Resumen}

Introducción: La satisfacción del paciente oncológico con el equipo de salud es de relevancia para evaluar la calidad de la atención del sistema de salud. En México no se dispone de una herramienta válida y confiable para evaluar este constructo. Objetivo: Validar el Instrumento de Evaluación de Funcionalidad en el Tratamiento para Enfermedades Crónicas-Satisfacción con el Tratamiento-Satisfacción del Paciente (FACIT-TS-PS) versión 4, en pacientes mexicanos con cáncer. Método: Diseño transversal, muestreo no probabilístico, por disponibilidad. La muestra consistió en 200 pacientes diagnosticados con cáncer, con edad promedio de $45.86 \pm 15.01$ años. Se realizó un análisis factorial exploratorio y confirmatorio. Resultados: Se identificaron cuatro factores con un alfa de Cronbach de 0.945 y una varianza explicada de $68.15 \%$. El análisis factorial confirmatorio indicó que el modelo teórico propuesto se ajusta a los datos con error próximo a cero y que, además, es equilibrado y mide cuidadosamente la satisfacción global del paciente con el tratamiento. Conclusión: FACIT-TS-PS mostró ser un instrumento válido y confiable para su uso en la atención clínica e investigación dirigida a pacientes mexicanos con cáncer. Se recomienda su utilización en la evaluación de equipos de salud multidisciplinarios en oncología en México.

PALABRAS CLAVE: Cáncer. Satisfacción con el equipo de salud. Validación de un instrumento. Población mexicana.

\section{Validation of an instrument to assess health care quality (FACIT-TS-PS) in cancer patients}

\begin{abstract}
Introduction: Cancer patient satisfaction with the healthcare team is of great relevance for assessing the quality of the care provided by the health system. In Mexico, no valid and reliable tool is available to assess this construct. Objective: To validate the Functional Assessment of Chronic Illness Therapy-Treatment Satisfaction-Patient Satisfaction (FACIT-TS-PS) instrument, version 4, in cancer patients. Method: Cross-sectional design, non-probability convenience sampling. The sample consisted of 200 cancer-diagnosed patients, with mean age of $45.86 \pm 15.01$ years. Exploratory and confirmatory factor analyses were conducted. Results: The exploratory factor analysis identified four factors, with a Cronbach alpha of 0.945 , and an explained variance of $68.15 \%$. The confirmatory factor analysis indicated that the proposed theoretical model adjusts to the data with an error close to zero and, in addition, it is balanced and carefully measures overall patient satisfaction with the treatment. Conclusion: FACIT-TS-PS was shown to be a valid and reliable instrument for use in clinical care and research in Mexican cancer patients. Its use is recommended in the evaluation of oncology multidisciplinary healthcare teams in Mexico.
\end{abstract}

KEY WORDS: Cancer. Satisfaction with the health care team. Validation of an instrument. Mexican population.

Correspondencia:

*Oscar Galindo-Vázquez

E-mail: psigalindo@yahoo.com.mx
Gac Med Mex. 2020;156:405-412

Disponible en PubMed

www.gacetamedicademexico.com

0016-3813/@ 2020 Academia Nacional de Medicina de México, A.C. Publicado por Permanyer. Este es un artículo open access bajo la licencia CC BY-NC-ND (http://creativecommons.org/licenses/by-nc-nd/4.0/). 


\section{Introducción}

La satisfacción del paciente con la atención médica es una variable multidimensional, en la cual el individuo evalúa distintos aspectos de la atención médica. ${ }^{1}$ Incluye inquietudes sobre la enfermedad y su tratamiento, carga financiera, comunicación con el equipo de salud, acceso a los servicios, satisfacción con las explicaciones del tratamiento y confianza en su médico. ${ }^{2}$ Es particularmente relevante en el campo de la atención del cáncer, caracterizada por importante cantidad de consultas médicas, estancias prolongadas en hospitalización y variedad de tratamientos. La satisfacción generada por aspectos interpersonales en la interacción con el personal médico o de enfermería determina, en gran medida, la satisfacción global del paciente oncológico con el proceso de atención médica ${ }^{3-9}$ y repercute en la adherencia terapéutica, ${ }^{10,11}$ las alteraciones psicológicas, ${ }^{12}$ la calidad de vida ${ }^{13,14} \mathrm{e}$, incluso, en una mayor supervivencia. ${ }^{11}$

La satisfacción del paciente es apreciada como relevante al menos en cuatro áreas: comparación de sistemas o programas de salud, evaluación de la calidad de los servicios de salud, identificación de los aspectos que necesitan modificación en los servicios y asistencia a las organizaciones sociales en la identificación de consumidores con poca aceptabilidad de los servicios..$^{15}$ La evaluación de la satisfacción se convierte en una tarea permanente y dinámica que aporta datos de cómo se encuentra y qué falta para cumplir con las expectativas de los pacientes. No solo permite obtener un indicador de excelencia, es más un instrumento de la excelencia. ${ }^{16}$

Para el sector de salud mexicano es fundamental contar con un instrumento que proporcione información válida y confiable respecto a la evaluación que realiza el paciente del proceso de atención médica. En este contexto, algunos instrumentos que se han utilizado para medir la satisfacción del paciente con la atención médica son CASE-cancer, ${ }^{17}$ ChPSQ-9, ${ }^{18}$ EORTC IN-PATSAT $32^{19}$ y PSCC. ${ }^{20}$ Dichos instrumentos presentan una variabilidad considerable de constructos, la mayoría de ellos evalúa la satisfacción del paciente con el médico y omite diversos personajes importantes del equipo de salud. En la revisión de la literatura no se encontró una escala validada para población mexicana con cáncer que evaluara la satisfacción del paciente con el equipo de salud.

Por lo tanto, el objetivo de este estudio fue validar el Instrumento de Evaluación de Funcionalidad en el Tratamiento para Enfermedades Crónicas-Satisfacción con el Tratamiento-Satisfacción del Paciente (FACIT-TS-PS) versión 4, capaz de evaluar la satisfacción del paciente enfocándose en la confianza y claridad de la comunicación que se tiene con tres de los principales grupos de atención médica oncológica: personal médico, personal de enfermería y personal encargado del tratamiento (asistentes técnicos, laboratoristas clínicos).

\section{Método}

Se solicitó la autorización a la organización FACIT.org para validar en México el instrumento. La investigación fue aprobada por el Comité de Ética y el Comité de Investigación del Instituto Nacional de Cancerología de la Ciudad de México.

La obtención de los participantes fue por disponibilidad en los servicios ambulatorios del Instituto Nacional de Cancerología, entre octubre de 2018 y diciembre de 2018. Los participantes aceptaron participar en la investigación previa comprensión y firma del consentimiento informado. Los criterios de participación fueron los siguientes:

- Criterios de inclusión: diagnóstico de cualquier tipo de cáncer, en cualquier tipo de tratamiento o periodo de seguimiento, y saber leer y escribir.

- Criterios de exclusión: deterioro cognitivo que impidiera contestar las encuestas o deficiencia visual o auditiva severa.

- Criterio de eliminación: no contestar de forma completa el instrumento y llenado incorrecto de los cuestionarios.

A 15 expertos que forman parte de equipos multidisciplinarios en oncología se les envió FACITSTS-PS y un formato para evaluar criterios de pertinencia, redacción, lenguaje apropiado para la población, validez teórica, validez aparente y validez de contenido. Posteriormente, se realizaron modificaciones para mejorar el entendimiento de las instrucciones y reactivos.

La adaptación al español en población mexicana se realizó mediante una prueba piloto en 29 pacientes. Se aplicó un formato de registro de caso de acuerdo con los lineamientos de Mora et al., que incluyó un cuestionario específico sobre la comprensión de las instrucciones, cada reactivo y las opciones de respuesta. ${ }^{21}$

Se diseñó una cédula de identificación del participante que incluía datos sociodemográficos y clínicos. Se empleó FACIT-TS-PS versión 4, desarrollado por Peipert et al., ${ }^{2}$ el cual constituye una escala tipo Likert autoadministrada que permite evaluar la satisfacción 
del paciente con el equipo de salud, conformada por 26 reactivos distribuidos en cinco factores: comunicación médica (alfa $=0.95)$, comunicación con el personal de tratamiento (alfa $=0.89$ ), competencia técnica (alfa $=0.86$ ), comunicación con enfermería (alfa $=0.72$ ) y confidencialidad y confianza (alfa $=0.93$ ); así como por tres reactivos individuales que no se incluyen en la sumatoria final. En total se trató de 29 reactivos.

Los datos fueron analizados con el programa estadístico SPSS versión 21. Se empleó un análisis factorial de componentes principales con rotación varimax en el que los criterios utilizados fueron los siguientes: sin suprimir pequeños coeficientes, no tomar un mínimo de reactivos por factor y coeficientes de consistencia interna por cada factor alfa de Cronbach $\geq 0.60$.

Se evaluó el ajuste del modelo a cuatro factores mediante un análisis factorial confirmatorio, en el que se uso el método de máxima verosimilitud que incluyó los pasos siguientes:22-24 identificación y especificación del modelo, estimación de parámetros estandarizados (correlaciones $\mathrm{R}^{2}$, covarianzas, índices de modificación y proporciones críticas de las diferencias) y, por último, evaluación del ajuste mediante la observación de límites aceptables de los estimadores, así como no colinealidad en las variables medidas.

Se estimaron los siguientes índices: $\chi^{2}$, razón de $\chi^{2} /$ grados de libertad, índice de bondad de ajuste (goodness of fit index, GFI) y sus complementos: el índice de Tucker-Lewis (Tucker-Lewis index, TLI) y el índice de bondad de ajuste corregido (adjusted goodness of fit index, AGFI); así como el índice de bondad de ajuste comparativo (comparative fit index, $\mathrm{CFI}$ ), ${ }^{25}$ que constituye el mejor indicador para muestras iguales 0 superiores a 200 y la raíz cuadrada media del error de aproximación (root mean square error of approximation, RMSEA).

\section{Resultados}

Se estudió una muestra no probabilística de 200 pacientes de ambos sexos con diagnóstico de cáncer, con un promedio de $45.86 \pm 15.01$ años, que acudieron a consulta al Instituto Nacional de Cancerología (Tabla 1).

El valor de la prueba de Kaiser-Meyer-Olkin fue de 0.91 ( $p=0.001$ ), lo que confirmó que la muestra fue adecuada para el análisis. El análisis factorial exploratorio mediante el método de componentes principales y rotación varimax con 26 reactivos arrojó un modelo con cuatro factores.
Los reactivos "¿Confió en las sugerencias de tratamiento de su(s) médico(s)?" (TS37) y “¿Tuvo confianza en su(s) médico(s)?" (TS36) del factor Confianza y confidencialidad cambiaron al factor Conocimientos y habilidades médicas; así como el reactivo "¿El personal a cargo del tratamiento respetó su privacidad?" (TS35) al factor Comunicación con el personal de enfermería. Se eliminaron los reactivos "¿Le pareció que el personal a cargo del tratamiento respondió a sus preguntas con sinceridad?" (TS34) y "¿Su(s) médico(s) le explicó (explicaron) los posibles beneficios de su tratamiento?" (TS10), debido a que tenía una carga factorial por encima de 0.40 en más de un factor.

En el análisis de confiabilidad se obtuvo un alfa total de Cronbach de 0.94 y 24 reactivos agrupados en cuatro factores que explicaron $68.15 \%$ de la varianza. El factor Confianza y confidencialidad se unificó con el factor conocimientos y habilidades médicas, por lo que se decidió nombrar a este nuevo factor como conocimientos médicos y confianza (Tabla 2).

Los índices relativos de bondad de ajuste comparativo (CFI, TLI y $\mathrm{AGFI}$ ) estuvieron muy cercanos al valor ideal (igual, próximo o mayor a 0.95 , respectivamente), lo que confirmó que se trata de un modelo aceptable en comparación con el modelo nulo. El valor $\mathrm{CFI}$, que tendió a 1 (superior a 0.5 ), señala un modelo más eficiente que el nulo. ${ }^{26}$

La proximidad a cero del índice de la raíz del residuo cuadrático promedio (RMR) e inferior a 0.08 $(R M R=0.044)$ ratifica la diferencia virtualmente nula entre la matriz de covarianza observada y la predicha; por lo tanto, puede asumirse que la discrepancia entre el modelo propuesto y los datos reales fue casi nula o muy baja. ${ }^{24}$

De forma general, el valor de chi cuadrada ajustado a 244 grados de libertad fue de 2.431 (es ideal que sea $<3$ ), con valor de $p<0.05$, lo que confirmó el ajuste absoluto del modelo a los datos observados. ${ }^{24}$

Se observó un valor cercano a cero en el índice RMSEA (0.085) y el intervalo superior fue muy cercano de 0.08 (0.076-0.093), lo que indicó casi nulidad en el error del modelo. ${ }^{24}$

En la prueba de Holter ( $n=101, p=0.01$ ), el tamaño de muestra teórico superó el utilizado, por lo que se pudo afirmar que el modelo era correcto y se aceptó la hipótesis (probabilidad de un error alfa de 0.01) de que la muestra era suficiente para los análisis. La Tabla 3 y la Figura 1 del modelo final resumen estos resultados. 
Tabla 1. Características sociodemográficas y clínicas de una muestra de 200 pacientes diagnosticados con cáncer

\begin{tabular}{|c|c|c|c|c|c|}
\hline \multicolumn{6}{|c|}{ Edad en años: rango 17-92, mediana 47 (36-56) } \\
\hline Variable & $n$ & $\%$ & Variable & n & $\%$ \\
\hline $\begin{array}{l}\text { Sexo } \\
\text { Mujer } \\
\text { Hombre }\end{array}$ & $\begin{array}{c}129 \\
71\end{array}$ & $\begin{array}{l}64 \\
36\end{array}$ & $\begin{array}{l}\text { Paternidad } \\
\text { Sí } \\
\text { No }\end{array}$ & $\begin{array}{c}141 \\
59\end{array}$ & $\begin{array}{l}70 \\
29\end{array}$ \\
\hline $\begin{array}{l}\text { Estado civil } \\
\text { Soltero(a) } \\
\text { Casado(a) } \\
\text { Unión libre } \\
\text { Otro (viudo, divorciado) }\end{array}$ & $\begin{array}{l}74 \\
83 \\
22 \\
21\end{array}$ & $\begin{array}{l}37 \\
41 \\
11 \\
10\end{array}$ & $\begin{array}{l}\text { Número de hijo(a)s } \\
1 \\
2 \\
3 \\
\geq 4\end{array}$ & $\begin{array}{l}32 \\
59 \\
28 \\
22\end{array}$ & $\begin{array}{l}16 \\
29 \\
14 \\
11\end{array}$ \\
\hline $\begin{array}{l}\text { Comorbilidad } \\
\text { Sí } \\
\text { No }\end{array}$ & $\begin{array}{c}54 \\
146\end{array}$ & $\begin{array}{l}27 \\
73\end{array}$ & $\begin{array}{l}\text { Ocupación } \\
\text { Empleado(a) } \\
\text { Autoempleado(a) }\end{array}$ & $\begin{array}{l}46 \\
37\end{array}$ & $\begin{array}{l}23 \\
19\end{array}$ \\
\hline $\begin{array}{l}\text { Tipo de comorbilidad } \\
\text { Diabetes }\end{array}$ & 10 & 5 & Hogar & $\begin{array}{l}49 \\
68\end{array}$ & $\begin{array}{l}24 \\
34\end{array}$ \\
\hline Hipertensión & 14 & 7 & Nivel de estudios & & \\
\hline $\begin{array}{l}\text { Diabetes + hipertensión } \\
\text { Infección por VIH }\end{array}$ & $\begin{array}{c}12 \\
4\end{array}$ & $\begin{array}{l}6 \\
2\end{array}$ & $\begin{array}{l}\text { Primaria } \\
\text { Secundaria }\end{array}$ & $\begin{array}{l}29 \\
44\end{array}$ & $\begin{array}{l}15 \\
22\end{array}$ \\
\hline Otro & 14 & 7 & Preparatoria & 56 & 28 \\
\hline $\begin{array}{l}\text { Lugar de residencia } \\
\text { Ciudad de México }\end{array}$ & 96 & 48 & $\begin{array}{l}\text { Licenciatura } \\
\text { Posgrado }\end{array}$ & $\begin{array}{l}56 \\
15\end{array}$ & $\begin{array}{c}28 \\
7\end{array}$ \\
\hline Provincia & 104 & 52 & $\begin{array}{l}\text { Nivel socioeconómico } \\
1\end{array}$ & 33 & 16 \\
\hline $\begin{array}{l}\text { Apoyo social } \\
\text { Bajo } \\
\text { Mediano } \\
\text { Alto }\end{array}$ & $\begin{array}{c}30 \\
57 \\
113\end{array}$ & $\begin{array}{l}15 \\
28 \\
56\end{array}$ & $\begin{array}{l}2 \\
3 \\
4 \\
5\end{array}$ & $\begin{array}{l}81 \\
62 \\
7 \\
6\end{array}$ & $\begin{array}{l}40 \\
31 \\
3 \\
3\end{array}$ \\
\hline $\begin{array}{l}\text { Índice de Karnofsky } \\
100\end{array}$ & 90 & 45 & 6 & 11 & 5 \\
\hline $\begin{array}{l}90 \\
80-60\end{array}$ & $\begin{array}{l}93 \\
17\end{array}$ & $\begin{array}{c}46 \\
8\end{array}$ & $\begin{array}{l}\text { Diagnóstico } \\
\text { Mama } \\
\text { Urología }\end{array}$ & $\begin{array}{l}55 \\
23\end{array}$ & $\begin{array}{l}27 \\
11\end{array}$ \\
\hline $\begin{array}{l}\text { Estadio } \\
\text { I } \\
\text { II } \\
\text { III } \\
\text { IV } \\
\text { Sin estadificar. }\end{array}$ & $\begin{array}{l}35 \\
41 \\
50 \\
44 \\
30\end{array}$ & $\begin{array}{l}17 \\
20 \\
25 \\
22 \\
15\end{array}$ & $\begin{array}{l}\text { Gastroenterología } \\
\text { Pulmón } \\
\text { Piel y partes blandas } \\
\text { Ginecología } \\
\text { Leucemia } \\
\text { Cabeza y cuello } \\
\text { Otro }\end{array}$ & $\begin{array}{l}21 \\
15 \\
24 \\
31 \\
13 \\
12 \\
6\end{array}$ & $\begin{array}{c}10 \\
7 \\
12 \\
15 \\
6 \\
6 \\
3\end{array}$ \\
\hline $\begin{array}{l}\text { Tratamiento } \\
\text { Seguimiento } \\
\text { Quimioterapia } \\
\text { Cirugía } \\
\text { Radioterapia } \\
\text { Quimioterapia-cirugía-radioterapia } \\
\text { Otro }\end{array}$ & $\begin{array}{l}45 \\
46 \\
32 \\
19 \\
33 \\
23\end{array}$ & $\begin{array}{c}23 \\
23 \\
16 \\
9 \\
16 \\
11\end{array}$ & & & \\
\hline
\end{tabular}

\section{Discusión}

La evaluación de la satisfacción del paciente oncológico con el equipo de salud es de gran relevancia debido a su relación con la continuidad terapéutica del paciente, los resultados en su salud y distintas afectaciones psicológicas. El instrumento FACIT-TS-PS presentó una estructura exploratoria similar a la versión original y propiedades psicométricas adecuadas. El análisis factorial confirmatorio indicó que el modelo teórico propuesto se ajustó casi perfectamente a los datos de la muestra utilizada y los indicadores estructurales del modelo (CFI, RMR y RMSEA) sugieren que se trata de un modelo con error próximo a cero, equilibrado y que mide cuidadosamente la satisfacción global del paciente con el equipo de salud. 
Tabla 2. Análisis factorial exploratorio de la escala FACIT-TS-PS versión 4 en pacientes con cáncer $(n=200)$

\begin{tabular}{|c|c|c|c|c|c|c|}
\hline $\begin{array}{l}\text { Alfa total de Cronbach }=0.94 \\
\text { Varianza explicada total }=68.15 \%\end{array}$ & & \multicolumn{3}{|c|}{ Carga factorial } & $\begin{array}{c}\overline{\mathrm{X}} \text { del } \\
\text { reactivo }\end{array}$ & $\begin{array}{l}\sigma \text { del } \\
\text { reactivo }\end{array}$ \\
\hline \multicolumn{7}{|c|}{ Factor 1. Comunicación con el médico } \\
\hline TS16. ¿Su(s) médico(s) parecía(n) entender sus necesidades? & 0.81 & 0.27 & 0.09 & 0.17 & 2.44 & 0.81 \\
\hline TS13. ¿Tuvo la oportunidad de decir lo que era importante para usted? & 0.80 & 0.11 & 0.16 & 0.19 & 2.38 & 0.911 \\
\hline TS15. ¿Su(s) médico(s) mostró (mostraron) un verdadero interés por usted? & 0.75 & 0.31 & 0.09 & 0.19 & 2.53 & 0.78 \\
\hline TS18. ¿Pudo hablar con su(s) médico(s) cuando lo necesitó? & 0.74 & 0.18 & 0.20 & 0.18 & 2.26 & 0.97 \\
\hline $\begin{array}{l}\text { TS27. ¿Lo/la motivaron a participar en la toma de decisiones respecto a su } \\
\text { atención médica? }\end{array}$ & 0.73 & 0.27 & 0.14 & 0.21 & 2.27 & 1.02 \\
\hline TS30. ¿Su(s) médico(s) parecía(n) respetar sus opiniones? & 0.68 & 0.29 & 0.26 & 0.25 & 2.52 & 0.80 \\
\hline TS14. ¿Su(s) médico(s) parecía(n) entender lo que era importante para usted? & 0.68 & 0.28 & 0.24 & 0.22 & 2.40 & 0.87 \\
\hline TS12. ¿Tuvo oportunidad de hacer preguntas? & 0.66 & 0.26 & 0.09 & 0.15 & 2.55 & 0.75 \\
\hline $\begin{array}{l}\text { TS28. ¿Tuvo suficiente tiempo para tomar decisiones respecto a su atención } \\
\text { médica? }\end{array}$ & 0.65 & 0.26 & 0.26 & 0.13 & 2.29 & 0.95 \\
\hline TS9. ¿Pudo entender las explicaciones de su(s) médico(s)? & 0.58 & 0.18 & 0 & 0.10 & 2.38 & 0.77 \\
\hline $\begin{array}{l}\text { TS11. ¿Su(s) médico(s) le explicó (explicaron) los posibles efectos } \\
\text { secundarios o riesgos de su tratamiento? }\end{array}$ & 0.41 & 0.38 & 0.07 & 0.34 & 2.44 & 0.86 \\
\hline
\end{tabular}

\section{Factor 2. Conocimientos médicos y confianza}

TS23. ¿Le pareció que su(s) médico(s) tenía(n) experiencia en el tratamiento de su enfermedad?

\begin{tabular}{|c|c|c|c|c|c|}
\hline 0.26 & 0.76 & 0.06 & 0.21 & 2.82 & 0.51 \\
\hline 0.30 & 0.75 & 0.21 & 0.03 & 2.77 & 0.52 \\
\hline 0.32 & 0.74 & 0.20 & 0.19 & 2.68 & 0.67 \\
\hline 0.27 & 0.69 & 0.09 & 0.25 & 2.70 & 0.65 \\
\hline 0.35 & 0.67 & 0.15 & 0.17 & 2.73 & 0.60 \\
\hline
\end{tabular}

TS37. ¿Confió en las sugerencias de tratamiento de su(s) médico(s)?

\section{Factor 3. Comunicación con el personal de enfermería}

TS32. ¿El personal de enfermería mostró un verdadero interés por usted?

$0.11 \quad 0.07$

TS33. ¿El personal de enfermería parecía entender sus necesidades?

$0.16 \quad 0.05$

TS31. ¿Pudo entender las explicaciones del personal de enfermería?

$0.13 \quad 0.18$

$\mathbf{0 . 8 1} 0.11$

TS35. ¿El personal a cargo del tratamiento respetó su privacidad?

0.28

0.32

0.62

0.02

2.74

TS21. ¿El personal a cargo del tratamiento le explicó la manera en que su salud y el tratamiento podrían afectar sus relaciones personales (familiares, amistades, laborales)?

TS20. ¿El personal a cargo del tratamiento le explicó la manera en que su salud y el tratamiento podrían afectar sus actividades diarias habituales (por ejemplo, bañarse, vestirse)?

TS22. ¿El personal a cargo del tratamiento le explicó la manera en que su salud y el tratamiento podrían afectarlo(a) emocionalmente?

TS19. ¿El personal a cargo del tratamiento le explicó la manera en que su salud y el tratamiento podrían afectar su trabajo habitual (incluidos los quehaceres domésticos)?

\begin{tabular}{|l|l|l|l|l|l|}
\hline 0.24 & 0.11 & 0.15 & $\mathbf{0 . 8 5}$ & 1.83 & 1.20 \\
\hline 0.18 & 0.22 & 0.24 & $\mathbf{0 . 8 0}$ & 2.12 & 1.13 \\
\hline 0.26 & 0.12 & 0.07 & $\mathbf{0 . 7 8}$ & 1.9 & 1.2 \\
\hline & & & & & \\
\hline
\end{tabular}

(Continúa) 
Tabla 2. Análisis factorial exploratorio de la escala FACIT-TS-PS versión 4 en pacientes con cáncer $(\mathrm{n}=200)$ (Continuación)

\begin{tabular}{|c|c|c|c|c|c|c|}
\hline $\begin{array}{l}\text { Alfa total de Cronbach }=0.94 \\
\text { Varianza explicada total }=68.15 \%\end{array}$ & \multicolumn{4}{|c|}{ Carga factorial } & $\begin{array}{c}\bar{X} \text { del } \\
\text { reactivo }\end{array}$ & $\begin{array}{l}\sigma \text { del } \\
\text { reactivo }\end{array}$ \\
\hline Valor alfa del factor & 0.93 & 0.88 & 0.86 & 0.88 & & \\
\hline Porcentaje de varianza explicada & 25.83 & 15.70 & 13.51 & 13.09 & & \\
\hline Media & 26.43 & 13.70 & 10.43 & 8.01 & & \\
\hline Desviación estándar & 7.42 & 2.49 & 2.31 & 4.00 & & \\
\hline Varianza del factor & 55.13 & 6.22 & 5.35 & 16.06 & & \\
\hline Correlación intraclase del factor & 0.56 & 0.60 & 0.61 & 0.66 & & \\
\hline Valor inferior & 0.50 & 0.55 & 0.55 & 0.60 & & \\
\hline Valor superior & 0.61 & 0.66 & 0.68 & 0.71 & & \\
\hline Valor F & 15.01 & 8.80 & 7.50 & 8.88 & & \\
\hline Valor $p$ & $\leq 0.001$ & $\leq 0.001$ & $\leq 0.001$ & $\leq 0.001$ & & \\
\hline
\end{tabular}

Tabla 3. Índices de bondad de ajuste del modelo confirmatorio resultante de la escala FACIT-TS-PS (satisfacción con el equipo de salud), 4 factores en pacientes con cáncer $(n=200)$

\begin{tabular}{|c|c|c|c|}
\hline Estadístico & Criterio deseable & Valor en el presente estudio & Interpretación \\
\hline $\begin{array}{l}\text { Ajuste absoluto } \\
\text { razón de } \chi^{2} / \text { grados de libertad }\end{array}$ & $<203$ & $595.772 / 244 \mathrm{gl}=2.441$ & $\begin{array}{l}\text { Los errores del modelo son nulos con la } \\
\text { muestra utilizada y el ajuste absoluto es } \\
\text { excelente }\end{array}$ \\
\hline $\begin{array}{l}\text { Índice de bondad de ajuste } \\
\text { (GFI) }\end{array}$ & $\begin{array}{l}>0.90 \\
\text { Preferente > } 0.95\end{array}$ & $\mathrm{GFI}=0.809$ & Ajuste aceptable \\
\hline $\begin{array}{l}\text { Índice de bondad de ajuste } \\
\text { comparativo (CFI) }\end{array}$ & $\begin{array}{l}>0.90 \\
\text { Preferente }>0.95\end{array}$ & $\mathrm{CFI}=0.900$ & Ajuste comparativo muy aceptable \\
\hline $\begin{array}{l}\text { Raíz del residuo cuadrático medio } \\
\text { (RMR) }\end{array}$ & Próximo a cero & $\mathrm{RMR}=0.044$ & $\begin{array}{l}\text { Error del modelo próximo a cero, casi ajuste } \\
\text { perfecto del modelo a los datos }\end{array}$ \\
\hline $\begin{array}{l}\text { Raíz del residuo cuadrático medio } \\
\text { de aproximación (RMSEA) }\end{array}$ & $\begin{array}{l}<0.08 \text {, próximo a } \\
\text { cero }\end{array}$ & RMSEA $=0.085(0.076-0.093)$ & $\begin{array}{l}\text { Error del modelo próximo a cero, casi ajuste } \\
\text { perfecto del modelo a los datos }\end{array}$ \\
\hline
\end{tabular}

El factor confianza y confidencialidad evalúa la confianza en general del paciente con el equipo de salud. Al unificar los reactivos de este factor con el de conocimientos y habilidades médicas se identificó que la confianza en general con el equipo de salud recayó mayormente en la percepción de la experiencia del equipo médico en el tratamiento, lo cual se podría explicar de acuerdo con lo que indica Wright, quien menciona que la confianza en la experiencia de los médicos es la principal preocupación de los pacientes con cáncer. Ser médico era suficiente, pero la confianza aumentaba cuando los médicos mostraban eficiencia y habilidades técnicas; ${ }^{9}$ incluso, los pacientes manifiestan tener confianza en la profesión médica como agente principal del sistema. Esta confianza es superior a la depositada en otras profesiones sanitarias y se mantiene a pesar de las quejas en torno a la atención sanitaria recibida. ${ }^{3}$ En el mismo sentido, Navarro menciona que los pacientes dan más importancia a la competencia del médico, la información sobre la enfermedad y los tratamientos, que a los aspectos de apoyo emocional y contextual. ${ }^{27}$

De acuerdo con la muestra obtenida en esta investigación, $40 \%$ de los participantes tenía un nivel socioeconómico 2, es decir, bajo. Se ha encontrado que las poblaciones de menores ingresos tienen menores niveles de satisfacción, sobre todo las que asisten a hospitales públicos. ${ }^{28}$ De los participantes, 


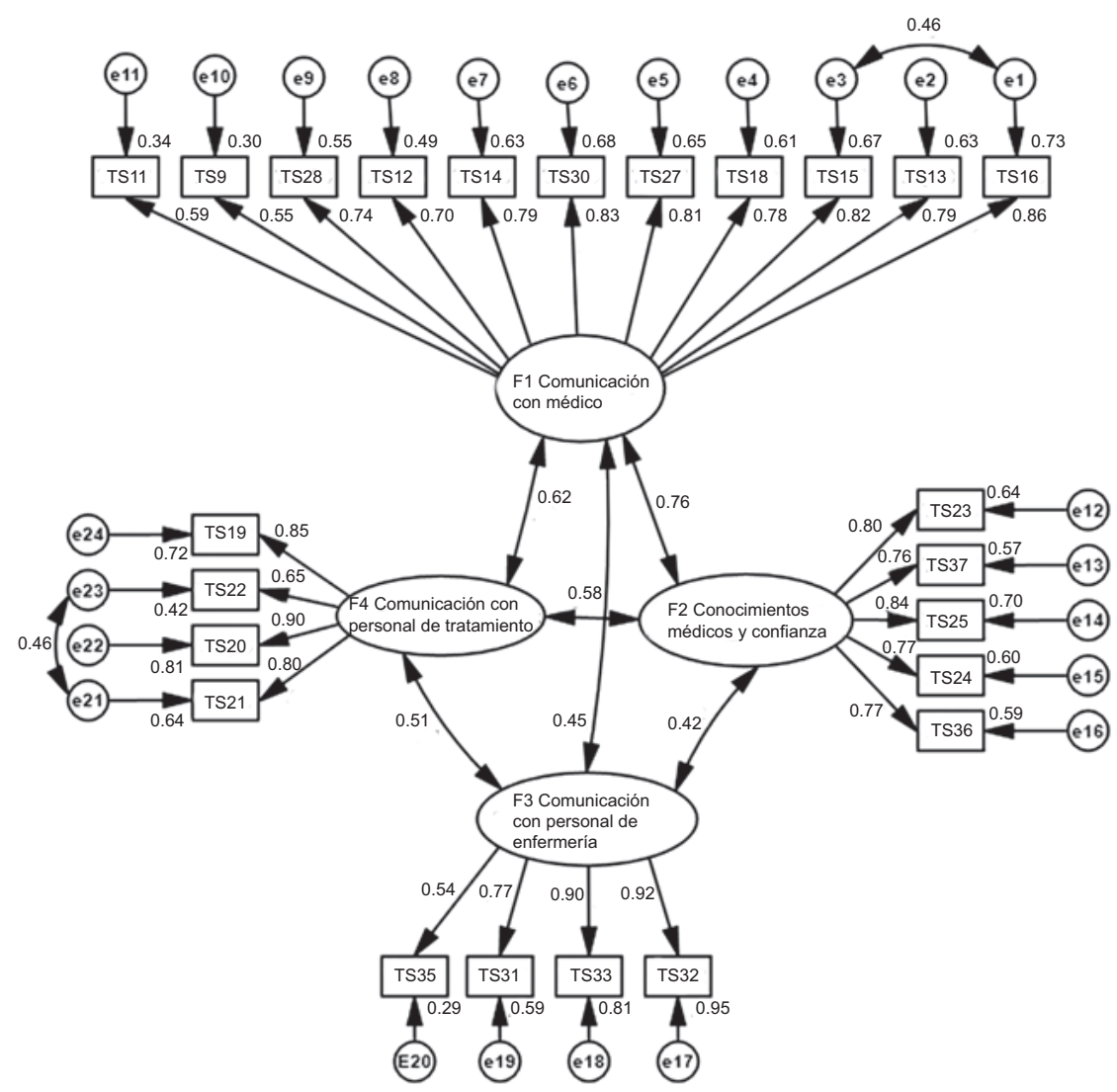

Figura 1. Modelo de análisis factorial confirmatorio de primer orden de cuatro factores de FACIT-TS-PS versión 4. $\chi^{2}=595.772,244$ gl, razón de $\chi^{2}$ grados de libertad $=2.431, p=0.000$, índice de bondad de ajuste comparativo $=0.900$, índice de Tucker-Lewis $=0.887$, índice de bondad de ajuste corregido $=0.766$, raíz del residuo cuadrático medio $=0.044$, raíz cuadrada media del error de aproximación $=0.085$ (0.076-0.093), prueba de Hoelter, $n=101(p=0.01)$.

$27 \%$ presentaba alguna otra comorbilidad con el cáncer, por lo cual la atención médica debe enfocarse a atender y comprender las afectaciones físicas y psicológicas producto de la interacción del cáncer con distintas enfermedades crónicas, como la diabetes y la hipertensión. Por ello, conocer la satisfacción del paciente oncológico en escenarios del sector salud público en México es de gran relevancia.

La ventaja del FACIT-TS-PS sobre otros instrumentos de satisfacción es que, más allá de conocer la cantidad de la información comunicada al paciente, se centra en conocer la claridad de la comunicación con el personal médico, integrando al personal de enfermería y al personal encargado del tratamiento, los cuales interactúan constantemente en la atención de los pacientes oncológicos. La comunicación efectiva ejerce una influencia positiva no solo en la salud emocional del paciente sino también en la resolución de los síntomas físicos, el estado funcional y fisiológico y el ajuste a la enfermedad. ${ }^{7}$ Por lo anterior, la evaluación de satisfacción que proporciona FACIT-TS-PS podría ser empleada en distintos servicios de atención médica oncológica del país, con ventajas en comparación con los resultados de otros estudios realizados en México acerca de satisfacción del paciente entre las que se encuentran las siguientes:

- Utiliza una escala válida y confiable para conocer la satisfacción con el equipo de salud.

- Fue validada en población oncológica.

- Se enfoca en conocer la calidad de la comunicación con tres de los principales sectores de la atención médica.

- Se consideran aspectos de la comunicación relacionados con la información brindada sobre el impacto psicológico y social esperados por la enfermedad; adicionalmente, puede servir como una medida de resultado de la calidad de la atención médica o del tratamiento. ${ }^{13,14}$

Entre las limitaciones de esta investigación se encuentra la falta de validez externa mediante correlaciones 
con medidas concurrentes. Finalmente, se espera que FACIT-TS-PS sea empleado en población clínica mexicana. Se recomienda que en estudios futuros se evalúe la estabilidad del instrumento a lo largo del tiempo y se desarrollen investigaciones que permitan analizar el impacto de la satisfacción del paciente con el equipo de salud mediante otros constructos como la adherencia, la aceptabilidad del tratamiento, la calidad de vida y la sintomatología de ansiedad o depresión.

\section{Conflicto de intereses}

Los autores declaran no tener conflicto de intereses alguno.

\section{Financiamiento}

Los autores no recibieron patrocinio para llevar a cabo este artículo.

\section{Responsabilidades éticas}

Protección de personas y animales. Los autores declaran que los procedimientos seguidos se apegaron a las normas éticas del comité de experimentación humana responsable y de acuerdo con la Asociación Médica Mundial y la Declaración de Helsinki.

Confidencialidad de los datos. Los autores declaran que han seguido los protocolos de su centro de trabajo sobre la publicación de datos de pacientes.

Derecho a la privacidad y consentimiento informado. Los autores declaran que en este artículo no aparecen datos de pacientes.

\section{Bibliografía}

1. Linder-Pelz S, Struening E. The multidimensionality of patient satisfaction with a clinic visit. J Community Health. 1985;10:42-54.

2. Peipert J, Beaumont J, Bode R, Cella D, Garcia S, Hahn E. Development and validation of the functional assessment of chronic illness therapy treatment satisfaction (FACIT TS) measures. Qual Lifi Res. 2013;23:815-824.

3. Joey A. J. El paciente del siglo XXI. Anales Sis San Navarra. 2006:29:85-90.

4. Kleeberg U, Tews J, Ruprecht T, Höing M, Kuhlmann A, Runge C. Patient satisfaction and quality of life in cancer outpatients: results of the PASQOC study. Supportive Care in Cancer. 2005;13:303-310.
5. Lis CG, Rodeghier M, Gupta D. Distribution and determinants of patient satisfaction in oncology: a review of the literature. Patient Prefer Adherence. 2009:287-304.

6. Macleod CJ. Verbal communication in nursing. En: Faulkner A (editor). Communication. Escocia: Churchill Livingstone; 1984.

7. Travado L, Grassi L, Gil F, Ventura C, Martins C. La comunicación médico-paciente en los oncólogos del sur de Europa: la influencia de la orientación psicosocial y el agotamiento. Rev Toxicomanias. 2005;44:15-24.

8. von Gruenigen VE, Hutchins JR, Reidy AM, Gibbons HE, Daly BJ, Eldermire EM, et al. Gynecologic oncology patients' satisfaction and symptom severity during palliative chemotherapy. Health Qual Life Outcomes. 2006;4:84.

9. Wright EB, Holcombe C, Salmon P. Doctors' communication of trust, care, and respect in breast cancer: qualitative study. BMJ. 2004;328:864.

10. Bui QUT, Ostir GV, Kuo YF, Freeman J, Goodwin JS. Relationship of depression to patient satisfaction: findings from the barriers to breast cancer study. Breast Cancer Res Treat. 2005;89:23.

11. Gupta D, Rodeghier M, Lis CG. Patient satisfaction with service quality in an oncology setting: implications for prognosis in non-small cell lung cancer. Int J Qual Health Care. 2013;25:696-703.

12. Wyshak G, Barsky AJ. Satisfacción y eficacia de la atención médica en relación con la ansiedad y la depresión. Gen Hosp Psychiatry. 1995:17:108-114

13. Pascoe GC. Patient satisfaction in primary health care: a literature review and analysis. Eval Program Plann. 1983;6:185-210.

14. Locker $D$, Dunt $D$. Theoretical and methodological issues in sociological studies of consumer satisfaction with medical care. Soc Sci Med. 1978;12:283-292.

15. Seclén PJ, Darras C Satisfacción de usuarios de los servicios de salud: factores sociodemográficos y de accesibilidad asociados: Perú, 2000. An Fac Med. 2005;66:127-141.

16. Massip-Pérez C, Ortiz-Reyes R, Llantá-Abreu M, Peña-Fortes M, Infante-Ochoa I. La evaluación de la satisfacción en salud: un reto a la calidad. Rev Cubana Salud Publica. 2008;34:1-10.

17. Wolf, M. S., Chang, C. H., Davis, T., Makoul, G. Development and validation of the Communication and Attitudinal Self-Efficacy scale for cancer (CASE-cancer). Patient Education and Counseling. 2005;57(3), 333-341.

18. Wong WS, Fielding R, Wong CM, Hedley AJ. Psychometric properties of the Ninelitem Chinese Patient Satisfaction Questionnaire (ChPSQ in Chinese patients with hepatocellular carcinoma. Psychooncology. 2008;17:292-299

19. Brédart A, Bottomley A, Blazeby JM, Conroy T, Coens C, D'Haese S, et al. An international prospective study of the EORTC cancer in-patient satisfaction with care measure (EORTC IN-PATSAT32). Eur J Cancer. 2005; $41: 2120-2131$

20. Jean-Pierre P. Fiscella K., Freund KM, Clark J, Darnell J, Holden A, et al. Structural and reliability analysis of a patient satisfaction with cancer-related care measure: A multisite patient navigation research program study. Cancer. 2011;117:854-861.

21. Mora RJ, Bautista AN, Natera G, Pedersen D. Adaptación cultural de instrumentos de medida sobre estigma y enfermedad mental en la Ciudad de México. Salud Mental. 2013;36:9-18.

22. Kline R. Principles and practice of structural equation modeling. EE. UU. Guilford; 2005.

23. George D, Mallery P. Using SPSS for Windows step by step: a simple guide and reference. EE. UU.: University of Michigan; 2001.

24. Byrne B. Structural equation modeling with AMOS: basic concepts, applications, and programming. EE UU.: Routledge; 2010.

25. Ullman JB. Structural equation modeling: reviewing the basics and moving forward. J Pers Assess. 2006;87:35-50.

26. Hu L, Bentler M. Fit indices in covariance structure modeling: sensitivity to underparameterized model misspecification. Psychological. 1998;3:424-453

27. Navarro E, Limonero JT, Maté J, Gómez-Romero MJ. Necesidades de comunicación e información en el paciente oncológico superviviente. Psicooncologia. 2010;7:127-141.

28. Guzmán MA Ramos CL, Castañeda SO, López-del Castillo SD, Gómez AA. Satisfacción de los usuarios de una unidad de medicina familiar. Rev Med Inst Mex Seguro Soc. 2006;44:39-45. 